\title{
The consideration of the turbulence influence on the gas explosion expansion in non-closed areas
}

\author{
Iurii Polandov ${ }^{1, *}$, Dmitry Korolchenko ${ }^{1}$ \\ ${ }^{1}$ Moscow State University of Civil Engineering, Yaroslavskoe shosse, 26, Moscow, 129337, Russia
}

\begin{abstract}
In the gas explosions simulation practice in non-closed areas there is a common opinion of burning rate increase at turbulization of initial gas mixture stream. The article shows that in conditions of turbulization there is no increase of the burn rate, but of the flame front area. A wording change of pressure rise reason does not lead to a change of the calculation results, but gives the opportunity to look at the problem differently and interpret a physical process correctly. In order to prove it there are results of the vibrational combustion modeling in terms of gas explosion in the cylinder with an opening on the side. The results show that along with the pressure fluctuation there is burning area size fluctuation that occur synchronous and with the same amplitude. The modeling basis is the system of differential equations, that describe the adopted in gas dynamics fundamental conservation laws, the equation of state, written in the so-called Euler's form. The system is supplemented with flame spread conditions. The system solution is based on the Russian method of the numerical approximation of this system-the large-particle method.
\end{abstract}

\section{Introduction}

Hitherto in the calculation of pressure during the gas explosion in non-closed vessels it is widely used [1-5] the way of its modeling (in terms of pressure "quasiconstancy") by means of using the mass balance equation in form:

$$
d M / d t=m 2-m 1,
$$

where $\mathrm{M}$ is gasses mass in a vessel, $\mathrm{kg}$; $\mathrm{t}$ - time, $\mathrm{c}$; $\mathrm{ml}$ - mass consumption through the dumping slot, $\mathrm{kg} / \mathrm{sec} ; \mathrm{m} 2$ - mass income of the combustion products with initial mixture substitution, $\mathrm{kg} / \mathrm{sec}$.

Anyway, with all the variables in this equation much is clear, except the mass of products consumption- $\mathrm{m} 2$,. Concerning the $\mathrm{m} 2$ definition many researchers face the need of knowing the flame front area, as

$$
m 2=\rho u F,
$$

\footnotetext{
${ }^{*}$ Corresponding author: ikbs@mgsu.ru
} 
where $\rho$ - initial mixture density, $\mathrm{kg} / \mathrm{M} 3 ; \mathrm{u}$ - normal burning rate, $\mathrm{m} / \mathrm{sec} ; \mathrm{F}$ - flame front area, $\mathrm{m} 2$.

The exponent of gases density (initial mixture and consumption products) and its dependence on pressure and temperature can be described by the famous gas dynamic functions and the quantity of the burning rate that can be considered as equal to normal adjusted to the initial mixture temperature influence and the pressure in the vessel. It is quite another matter to determine the size of the flame front is in each moment of time. It is more complicated.

Though in one case it is possible to do. It is about a so-called central explosion; the steam-and-gas mixture is lit in the geometrical center of spherical vessel. However it is about vessels, that have diameter under $40 \mathrm{~cm}$, otherwise it can be affected by Archimedes' forces. Such explosion has purely informative exponent and does not occur practically.

In other cases there have to be phenomenological models of flame area spread during explosion-there is no other way for calculations while having pressure quasiconstancy. According to publications, there is quite a lot of such models, that can be divided into two groups : the first ones describe the area spread while the process occurs "laminarly", it happens when the flame front is a thin layer of spherical or other form; and the other group considers the initial mixture turbulization. First group models are based on the considerable number of experimental data that is used to approximate the flame front spread. Considering the flame front deflection from the sphere comes to modification introduction to the equation (2). Naturally, in this case the calculation results from the equation will give similar exponents to the experimental data. This calculation option in terms of science is close to the so-called "vicious circle", when one of the fundamental parameters is taken from the experiment and is used later to describe the same experiment. It is close to simple result approximation. In this case, one can certainly argue about which one of the approximation models is more successful, though it is clear that the difference between them cannot have the constant antagonistic character. It should be acknowledged that such models are right in the initial conditions and do not have universal characteristics. Therefore trying to extend such models to the other more complicated flame front form, one can get considerable deflections from experimental results

In case of turbulent stream, the situation is much more difficult. It is known that in practice the stream turbulization leads to pressure rise, compared to the laminar flame front spread. This circumstance is for sure connected with the consumption products mass income increase $\mathrm{m} 2$. To consider it, one has to introduce modifications to product $\rho \mathrm{FF}$. Here comes a question, which of the multipliers from this product has to be corrected. After all being introduced to any of multipliers, the modification will not change the product result. In all known publications the modification is introduced to the burning rate $u$. The concept of "turbulent burning rate" is being also introduced [4-8].

There are no arguments given, except the fact that there is an option when one has same calculation results, which is incorrect.

\section{Working hypothesis}

Everyone agreed with the statement of Russian scientist Mihelson (published by Raushenbakh B.V. [9]) that the burning rate possesses universal properties and serves as one of the intermolecular interaction result. Therefore it is not connected with gasdynamical continual process, at any case considering a deflagrational explosion. Such rate is considered normal, as it is always directed perpendicularly to the flame front. The flame spread process certainly cannot be mixed with the gas volume expansion process due to a chemical reaction. The gas expansion occurs in the flame front, which is a part of the initial mixture with a normal rate. 
The stream turbulization is a gasdynamical process on a continuum and cannot influence the normal burning rate, which occurs on a molecular scale. According to common concepts the visible flame spread on an initial mixture consists of three components: flame spread rate as a plunger, which compresses the initial mixture, the front movement to the dumping slot and normal burning rate. The formed vortexes carry a flame front part to a rotation on their exterior surface, they "deliver" its centers on the volume. Splitting up to smaller vortexes, by virtue of the natural vortex attenuation process and increasing its surface, they transfer the surface burning, according to visual feelings, to a volumetric one. Should be noted that the burning occurs with the same normal rate even in the small vortex. The burning area expands due to the turbulization. Naturally, while estimating the turbulence influence one should focus on well-known works on this subject, however it should be stressed that there are a few calculation methods in this field of knowledge. Unfortunately, in our case there is a gas expansion inside the vortexes, which is being superimposed to the turbulent stream while burning, which seriously influence their situation. Here comes the only solution to make and experiment and to introduce modifications to the exponents of one of the co-multipliers in the product $\rho u F$.

It should be noted that while introducing the modifications to the rate exponent, one uses the normal burning rate as a fundamental exponent, and takes a specific vessel area as a square exponent, e.g the total area of internal vessel sides or the area of its transverse section. In the offered option of modification introduction the normal burning rate is left unaltered, but the modifications are being introduced into the flame front area exponent, where the specific areas can be used as a fundamental exponent, which is the same as in the first option. One can also use the calculated flame front area in the vessel, without turbulization.

There is a calculative experiment data, which gives the opportunity to determine not only the pressure, but also the current flame front area exponent, and also makes it possible to understand the connections between them. This data is given as a proof that the turbulence influence the explosion pressure not through the burning rate increase, but through the flame front is expansion.

\section{Experiment facilities}

Mathematical model. The working hypothesis check is carried out through the numerical modeling of the gas explosion in the room with a slot, using the example of the model cylindrical chamber (figure 1). The task is to model environmental dynamics by using one of the methods of solving nonstationary multidimensional problems of the continuum mechanics (CFD).

1. Initial equation system.

There are famous gas dynamic differential equations in the Euler's form that signify the fundamental laws of conservation, included to the initial system: the equation of continuity, impulse and environmental energy: 


$$
\left\{\begin{array}{l}
\frac{\partial \rho}{\partial t}+\operatorname{div}(\rho U)=0 \\
\frac{\partial \rho U_{x}}{\partial t}+\operatorname{div}\left(U_{x} \rho U\right)+\frac{\partial p}{\partial x}=0 \\
\frac{\partial \rho U_{y}}{\partial t}+\operatorname{div}\left(U_{y} \rho U\right)+\frac{\partial p}{\partial y}=0 \\
\frac{\partial \rho U_{z}}{\partial t}+\operatorname{div}\left(U_{z} \rho U\right)+\frac{\partial p}{\partial z}=0 \\
\frac{\partial \rho B}{\partial t}+\operatorname{div}(\mathrm{E} \rho U)+\operatorname{div}(p U)=0 .
\end{array}\right.
$$

There is a gas law equation, closing the system

$$
p=\rho I(\gamma-1) \text {. }
$$

Where $\rho$ - gas density, $\mathrm{kg} / \mathrm{m} 3 ; \bar{U}$ - velocity vector, $\mathrm{m} / \mathrm{s} ; \mathrm{E}-$ specific total energy, $\mathrm{J} / \mathrm{kg}$; $\mathrm{p}$ - pressure, $\mathrm{Pa} ; \gamma$ - adiabatic index of the medium; I - specific internal energy, $\mathrm{J} / \mathrm{kg} ; \mathrm{Ux}$, $\mathrm{Uy}, \mathrm{Uz}$ - velocity components respectively along the axes $\mathrm{x}, \mathrm{y}, \mathrm{z}, \mathrm{m} / \mathrm{s}$.

2. The initial and boundary conditions.

It is assumed that all space is filled with a stoichiometric composition of methane-air mixture, the initial pressure and temperature of the mixture has a normal exponent. Boundaries of the room impermeable, in explosions gas is ejected through the window. The gas composition is not changed in the process is the same combustion and air. Window is a weightless and collapsing at the initial moment of the explosion.

3. The common principle of solving the equation system.

The numerical system solution is carried out through the large particles method, LPM, [10], developed by O.M. Belotserovskiy and Yu.M. Davydov. Along with other modern methods [11-14] the large particles method allows to analyze gasdynamic streams without aprioristic information about the solving structure.

There is Euler's (fixed) scale put on the range of integration, which consists of rectangular cells with the sides $\Delta \mathrm{x}, \Delta \mathrm{y}$ и $\Delta \mathrm{z}$. The calculation consists of multiple repetition of moves in time, where every move includes 3 phases:"euler's", "langrazhov's" one and the final calculation phase.

4. Consideration of the flame front expansion process.

To describe the flame front expansion process is used the approach, which harmoniously fits into the approximation system model. According to the approach there is an extra parameter of the cell state- mass fraction of combustion products $f$ in the cell [15]. This parameter is seen as the following ratio for every cell

$$
f=m_{B} / m \text {. }
$$

Where $\mathrm{m}$ - total mixture mass in the cell, $\mathrm{kg} ; \mathrm{m}_{\mathrm{B}}-$ products consumption mass in the cell, $\mathrm{kg}$.

The calculated cells are divided into three groups (including the calculation error $-\varepsilon$ ):

- the initial mixture cells, where the condition $f<\varepsilon$ is satisfied;

- «burnt» cells $-f>1-\varepsilon$;

- «burning» cells $\varepsilon<f<1-\varepsilon$.

The consideration of flame front spread condition while solving the equation system seriously worsens the solution stability, therefore one has to consider a time move, which 
satisfies the Kurant-Friedrichs-Levy's stability condition with a greater (more than 10 multiple) reserve.

Complete description of the model and used differential schemes is presented in [16]. It also shows the mathematical model adequacy.

\section{Calculating object}

There is a scheme of a conventional cylinder on the Figure 1, where the numerical experimental explosion is being carried out. Its length is $1500 \mathrm{~mm}$, and diameter $-200 \mathrm{~mm}$. The mixture of propane and air is used as a stoichiometric mixture. The ignition is always carried out at the same place, at the left-hand end. The dumping slot location is changeable and has a distance 11. In every slot position there was a change of its diameter. There were around 100 numerical experiments carried out in total. In the numerical model the cells sides are considered equal to $\Delta x=\Delta y=\Delta z=1 \mathrm{~cm}$, their total number is around 70000 , move in time is $\Delta \mathrm{t}=5 \cdot 10-7 \mathrm{c}$.

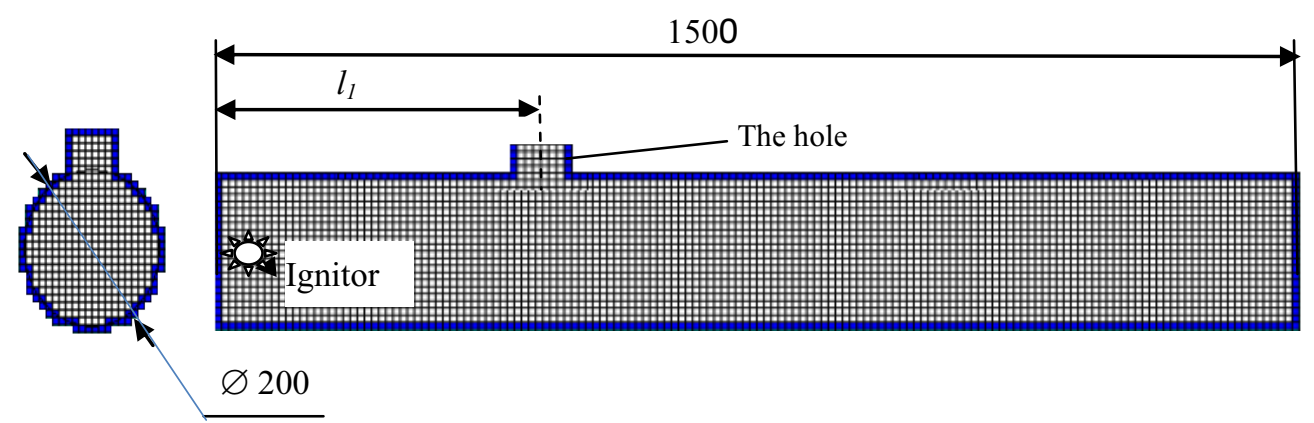

Fig. 1. Estimated area.

\section{Results of experiment}

With the distance 11 equal to $50 \mathrm{~mm}$ and the slot diameter $61 \mathrm{~mm}$ considerable pressure fluctations were dicovered during the explosion. There are individual shots of flame expansion inside the cylinder in this case on the Figure 2. 


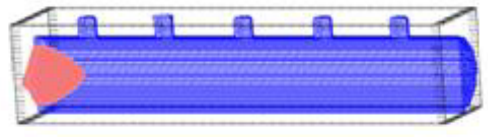

0.025

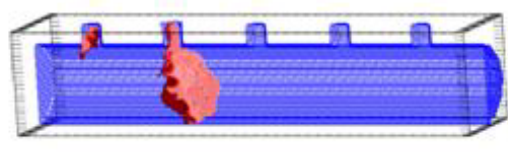

$0,075 \mathrm{~s}$

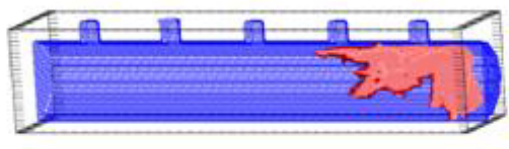

$0.225 \mathrm{~s}$

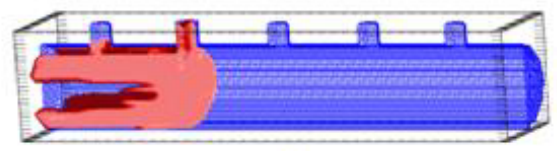

$0.05 \mathrm{~s}$

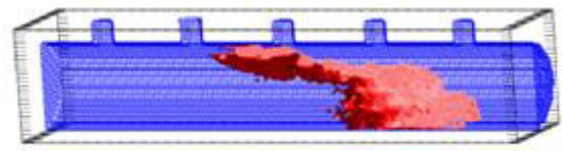

$0.15 \mathrm{~s}$

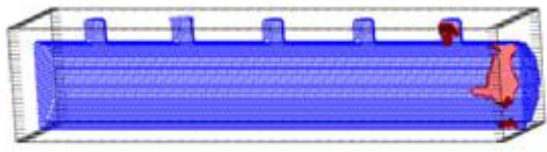

$0.325 \mathrm{~s}$

Fig. 2. The calculated flame front position at different time moments, $S l o t ~ l=50 \mathrm{~cm}$ and, $d=61 \mathrm{~mm}$.

There is a maximum pressure definition introduced to mark its existence areas in the experiment $\mathrm{p}_{\max }$ concerning maximal $\overline{\mathrm{p}}$, defined as $\overline{\mathrm{p}}=\mathrm{p}_{\max } / \mathrm{p}_{1 \max }$, where there is a exponent pmax 1 in denominator on the position $1_{1}=20 \mathrm{~cm}$. Using this exponent we drew a dependence graph $\overline{\mathrm{p}}=\overline{\mathrm{p}}$ (l) with fixed slot diameter exponents. There is a recalculation data results of the numerical experiment on the figure 3 . There is $\bar{p}$ put on the ordinate axis and the slot position put on the abscissa axis. In view of the pressure dependence on the slot position there is a notorious abnormality, which occurs as drastic pressure increase. It is possible with the $61 \mathrm{~mm}$ slot diameter and the slot position close to $1=50 \mathrm{~cm}$.

Should be considered (Figure 4) that the fluctuation frequency was changing with the flame front move from 100 to $200 \mathrm{~Hz}$. The fluctuation amplitude due to it reached the exponent of $20 \mathrm{kPa}$. The amplitude had maximal exponents with the frequency equal to 200 $\mathrm{Hz}$. The flame front area size changes synchronously (in phase) with pressure fluctuation, area changes have the same order as pressure changes.

\section{Numerical experiment data analysis}

The findings can be described the following way. Flame front size fluctuations lead to pressure fluctuations, as the product consumption mass income exponent fluctuates. The pressure fluctuations, because stagnant half-wave, are connected to longitudinal gas fluctuations, which are the most considerable in the middle part of the cylinder. The gas fluctuations at the same time fluctuate the flame front. This situation is clearly visible on the visual picture of explosion dynamics, where the fluctuation amplitude of the flame front position at the moment of pressure peak is equal to not less than $20 \mathrm{~cm}$. On the Figure 2 it is visible that the fluctuations starts as soon as the combustion products enter the dumping slot and continues during the time, until burning gas particles enter it. It is also possible, that the longitudinal gas fluctuations "swingle" and turbulizate the flame front, increasing burning cells number, which is the combustion product mass income. According to the Figure 4 the flame front area is $0.5 \mathrm{~m} 2$, which exceeds the cylinder cross-section area by 16 
times, which means it is a turbulent burning. On the Figure 2 it is visible, how the flame front thickens at the fluctuation period. There is an obvious and direct link between explosion pressure and flame front size, even with the turbulent burning option consideration. The therm "flame front area expansion in terms of turbulence" or shorter "turbulent flame area" seems to define the physical aspect of the gas explosion process more clearly.

It should be noted that in every numerical experiment the burning rate exponent was constant and in common sense depended only on the temperature and initial mixture pressure.

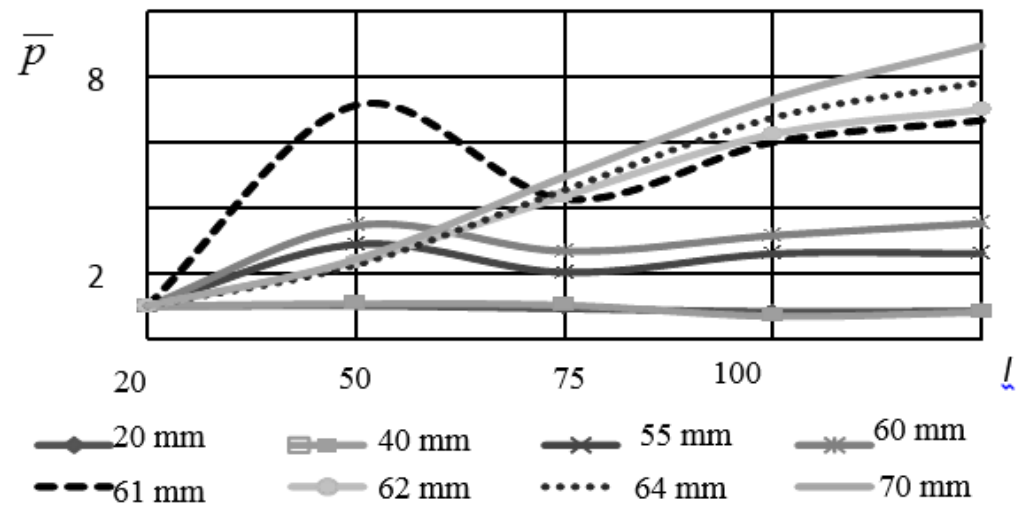

Fig. 3. Dependence of pressure of the explosion on the size and position of the tube relative units.

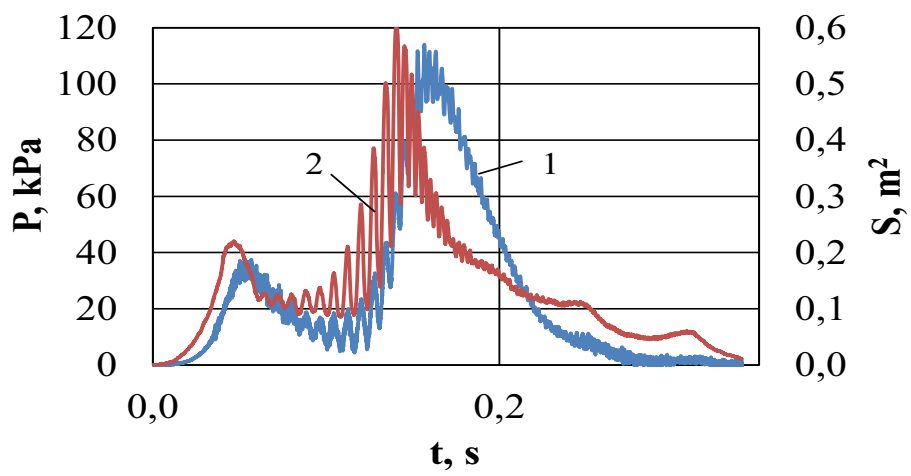

Fig. 4. Pressure dynamics in the chamber. (the slot is on the position 2, $d=60 \mathrm{~mm}$ )

Has to be stressed that there were no specific equations and dependences introduced to the system in order to stimulate auto-fluctuations. The reached effect is a product of the given equations system and its numerical solution option, which was possible in terms of equations nonlinearity and mathematical model adequacy. The well-known and widespread in the EU computer program FLACS, which was specially created for gas explosions description, was not able to prove the same, though the modeling was done by program developers [12].

\section{Conclusion}

Though the used term "turbulent burning rate" with the introduced modifications to normal burning rate to "turbulization" does not change the calculation results of explosion pressure 
in non-closed volume, in terms of methodology it shows the physical process sense incorrectly. The logical argumentations show that it should be about the flame front expansion. These arguments are proved by the numerical experiment results, where was defined the direct link between the pressure exponent and the flame front size during the turbulent burning. Therefore we can conclude that there is a need of using the term "turbulent flame area" while calculating the pressure.

\section{References}

1. V. Molkov, A. Baratov, A. Korolchenko, Progress in Astronautics and Aeronautics 154, 117-131 (1993)

2. R. Zalosh, Explosion venting data and modeling literature reviev (Firexplo, Quincy, 2002)

3. R. Lautkaski, Research Report 2(52), 23-28 (2009)

4. W. Yuebin, School of Municipal \& Environmental Engineering, 150090 (2007)

5. Han Yong Li, Tianjin Univercity and Springer Verlag 14, 434-440 (2008)

6. C.R. Bauwens, S.B. Dorofeev, Research Division 02062, 1151 (2008)

7. F. Taminini, J.L. Chaffee, Twenty-Fourth International Symposium on Combustion 1, 1845-1851 (1992)

8. V.V. Molkov, R.M. Eber, A.V. Grigorash, F. Tamanini, R. Dobashi, Journal of Loss Prevention in the Process Industries, 395-402 (2003)

9. B.V. Raushenbakh, Vibrational burning (Fizmatgiz, Moscow, 1961)

10. Yu.M. Davydov, Large-particle method (Kluver academic publishers, London 1990)

11. S.K. Godunov, Math. Sbornik 47, 271-306 (1969)

12. H.H. Pedersen, P. Middha, Modelling of Vented Gas Explosions in the CFD tool FLACS, www.aidic.it/cet

13. D. Korolchenko, S. Voevoda, MATEC Web of Conferences 86, 04056 (2016) DOI: $10.1051 / \mathrm{matec}$ onf $/ 20168604056$

14. D. Korolchenko, S. Voevoda, MATEC Web of Conferences 86, 04038 (2016) DOI:10.1051/matecconf $/ 20168604038$

15. Iu.H. Polandov, M.A. Barg, V.A. Babankov, Sofia publications, 82 ( 2011)

16. Iu.H. Polandov, M.A. Barg, V.A. Babankov, Sofia publications, 84 (2011) 